\title{
Carbon Nanostructures Production by AC Arc Discharge Plasma Process at Atmospheric Pressure
}

\author{
Shenqiang Zhao, ${ }^{1,2,3}$ Ruoyu Hong, ${ }^{2}$ Zhi Luo, ${ }^{2}$ Haifeng Lu, ${ }^{2}$ and Biao Yan ${ }^{1}$ \\ ${ }^{1}$ College of Material Science and Engineering, Tongji University, Shanghai 201804, China \\ ${ }^{2}$ Chemical Engineering and Material Science \& Key Laboratory of Organic Synthesis of Jiangsu Province, \\ College of Chemistry, Soochow University, SIP, Suzhou 215123, China \\ ${ }^{3}$ Modern Experiment and Technology Center, Anhui University, Hefei 230039, China \\ Correspondence should be addressed to Ruoyu Hong,rhong@suda.edu.cn and Biao Yan, yanbiao2002@yahoo.com.cn
}

Received 6 July 2011; Accepted 22 August 2011

Academic Editor: Yanqiu Zhu

Copyright ( $\odot 2011$ Shenqiang Zhao et al. This is an open access article distributed under the Creative Commons Attribution License, which permits unrestricted use, distribution, and reproduction in any medium, provided the original work is properly cited.

Carbon nanostructures have received much attention for a wide range of applications. In this paper, we produced carbon nanostructures by decomposition of benzene using AC arc discharge plasma process at atmospheric pressure. Discharge was carried out at a voltage of $380 \mathrm{~V}$, with a current of $6 \mathrm{~A}-20 \mathrm{~A}$. The products were characterized by scanning electron microscopy (SEM), high-resolution transmission electron microscopy (HRTEM), powder X-ray diffraction (XRD), and Raman spectra. The results show that the products on the inner wall of the reactor and the sand core are nanoparticles with 20-60 nm diameter, and the products on the electrode ends are nanoparticles, agglomerate carbon particles, and multiwalled carbon nanotubes (MWCNTs). The maximum yield content of carbon nanotubes occurs when the arc discharge current is $8 \mathrm{~A}$. Finally, the reaction mechanism was discussed.

\section{Introduction}

Carbon nanomaterials, which usually are carbon blacks (CBs), fullerenes, and carbon nanotubes, carbon fibres, have a privileged position in all applications related to nanotechnology. Current and potential applications of carbon nanomaterials cover all segments of the energy and materials sectors [1]. Carbon black belongs to the first nanomaterials produced on an industrial scale in the early 20th century [2]. Production of $\mathrm{CB}$ is about 8 million tons per year in the world. Fullerenes are considered a versatile building block in organic chemistry for molecular engineering and practical application (photovoltaics, medicine, etc.). Carbon fibres and CBs are large-scale products used as reinforcement structures in polymers and metals. Carbon nanotubes have been proposed for many applications, including electronic devices, field emitters, hydrogen storage, scanning microscope probe tips, and composites [3].

This work focused on CBs, fullerenes, and carbon nanotubes.
Historically, the synthesis of these nanocarbons is closely related to plasma processes, particularly arc discharge. Initial papers claiming fullerenes and CNT production to gram scale were based on it [4]. In addition, carbon nanotubes discovery was associated with microscopic characterization of samples produced by the arc technique [5]. However, $\mathrm{CB}$ and fullerenes are currently produced at industrial scale in flame reactors $[6,7]$. This technique, which is classified within the gas-phase synthesis method, consists of the continuous production of carbon nanostructures by employing the flame heat to initiate chemical reactions producing condensable monomers. The flame is usually produced by burning a fraction of the carbon precursor, usually a hydrocarbon. The principle of the plasma process consists in replacing the incomplete combustion by directly cracking the hydrocarbon into carbon black and hydrogen owing to an external electric energy supply. Plasma processes provide a reasonable alternative to flame aerosol technology due to their number of advantages with respect to combustion [8]. At first, plasma processes are allothermal: the energy input is 


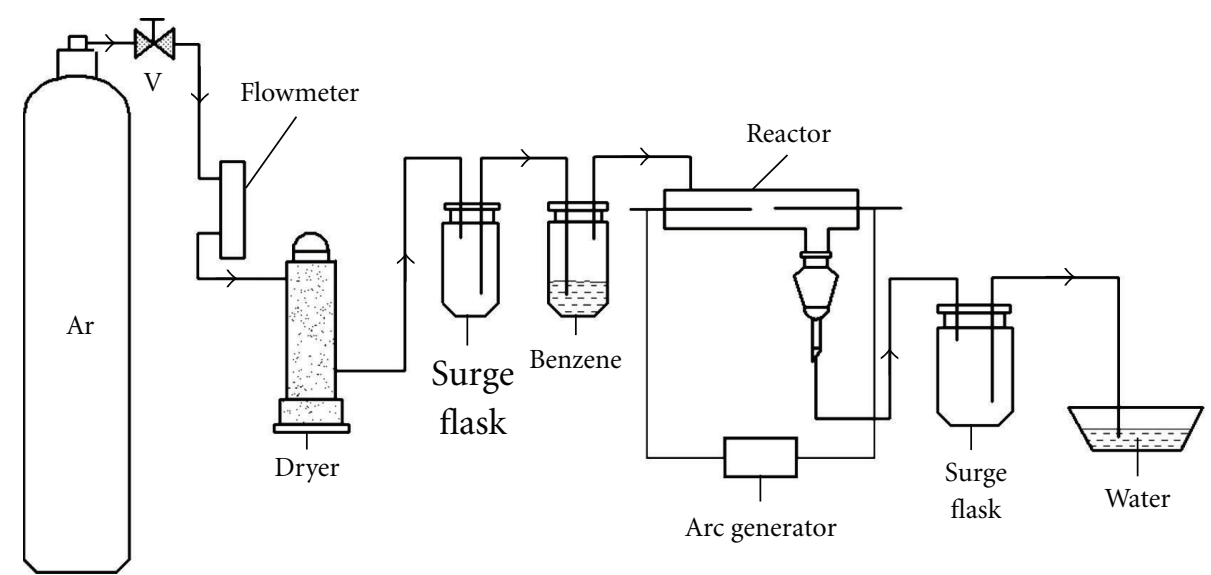

FIgURE 1: Schematic diagram of an AC arc plasma reactor system.

independent of chemical reactions happening in the reactor. Secondly, plasma techniques are usually environmentally clean processes since they are not a primary greenhouse gas emitter. Thirdly, the conversion efficiency is close to $100 \%$. Finally, hydrocarbon plasma conversion leads to the formation of hydrogen, a highly valuable by product considered today the most feasible energetic vector of the future.

In the conventional arc discharge processes used in producing fullerenes and carbon nanotubes, the electrodes are two graphite rods and the origin of the carbon precursor comes from the ablation of a graphite electrode. In this work, the two graphite electrodes were substituted with two tungsten electrodes, and the origin of the carbon precursor was the carbon black which comes from the decomposition of the benzene in the reactor. Benzene was used as the origin of the carbon precursor because the hydrocarbon (benzene, methane, acetylene, ethylene) has no influence on the products' properties [9] and the experimental device can be simplified.

\section{Experimental}

A schematic diagram of the carbon nanomaterials synthesis by benzene decomposition is shown in Figure 1. The thermal plasma was produced by an electric arc between tungsten electrodes with $6 \mathrm{~mm}$ in diameter connected to AC power supply, which allows a maximum power input of $8 \mathrm{~kW}$. The frequency of the electrical source is $50 \mathrm{~Hz}$. Argon (purity 99.99\%, Jinhong Gas Co., Ltd., China) was used as plasma gas, and benzene (AR, Sinopharm Chemical Reagent Co., Ltd., China) was used as carbon source. Benzene was carried into the reactor by argon flow. Plasma gas and benzene were injected simultaneously at atmospheric pressure with flow rates of $80 \mathrm{l} / \mathrm{h}$. Discharge was carried out at a voltage of $380 \mathrm{~V}$, with a current of $6 \mathrm{~A}, 8 \mathrm{~A}, 14 \mathrm{~A}$, and $20 \mathrm{~A}$, respectively.

In this experiment, the products were deposited on the internal wall of the reactor, the sand core, and the tungsten electrode ends. The products on the electrode ends showed radial, others evenly distributed on the surface of the reactor internal wall and sand core. These carbon nanostructures were characterized using a scanning electron microscopy
(S-4800, FE-SEM), a high-resolution transmission electron microscopy (JEM-2100, HRTEM-200 KV), powder X-ray diffraction (DX-2700, XRD), and laser Raman spectra (HR800, Raman). SEM was performed at $0.5-30 \mathrm{kV}$ with no metallisation of the samples. For TEM analysis, the collected products were first sonicated in alcohol. Then, the prepared suspension was deposited onto copper microgrids coated with amorphous lacey carbon membranes. Once dried, the microgrids were observed by HRTEM.

\section{Results and Discussions}

3.1. SEM and HRTEM Studies. A study conducted by SEM and TEM is conclusive to characterize the texture (elemental particles and their aggregating) as well as the structure (crystallinity) of the deposited carbon. It shows that the AC arc discharge plasma process is a versatile process for growing a wide range of carbon black.

SEM images were obtained on a film, deposited on a silicon wafer. Figures 2 and 3 show morphology of the sample obtained from benzene in argon plasma medium. Figures 2(a), 2(b), 2(c), and 2(d) illustrate the typical micrographs of the deposited carbon on the inner wall of the reactor and the sand core which correspond to the arc discharge current of $6 \mathrm{~A}, 8 \mathrm{~A}, 14 \mathrm{~A}$, and $20 \mathrm{~A}$, respectively. It was observed that the carbon particles exhibit a spherical particle with nanosized diameter. The diameter of the carbon particle varied from 20 to $60 \mathrm{~nm}$, and the mean diameter was about $30 \mathrm{~nm}$. Figures 3(a), 3(b), 3(c), and 3(d) illustrate the typical micrographs of the deposited carbon on the electrodes ends, which correspond to the arc discharge current of $6 \mathrm{~A}, 8 \mathrm{~A}, 14 \mathrm{~A}$, and $20 \mathrm{~A}$, respectively. It was clear that carbon nanotubes (CNTs), aggregate carbon nanoparticles, and agglomerate carbon particles are formed in all cases, and yield content of CNTs depends on the arc discharge current. The production rate of CNTs begins to increase and then decrease while the arc discharge current increased. The maximum, which is roughly about $80 \%$, was obtained while the current is up to $8 \mathrm{~A}$. The solid phase theory for carbon nanotubes may help us to understand the phenomena [10]. The temperature of the electrode ends needs to reach high temperature, on 


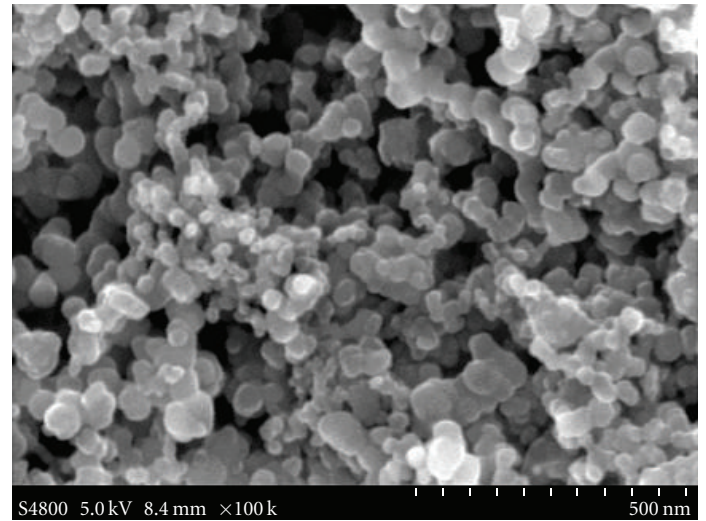

(a)

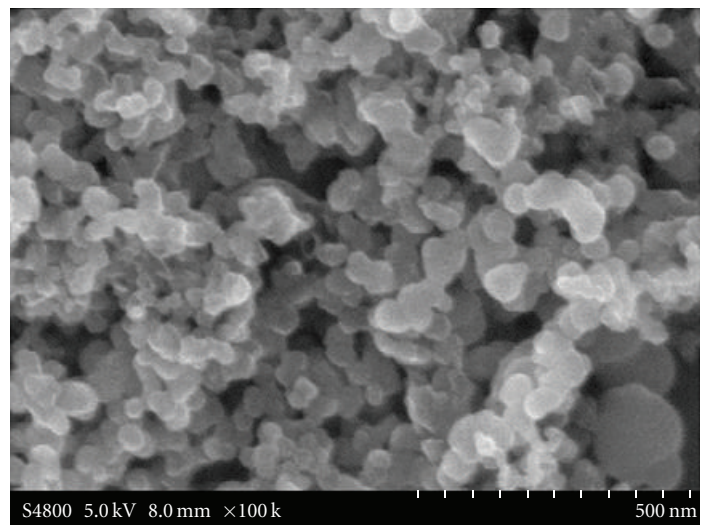

(c)

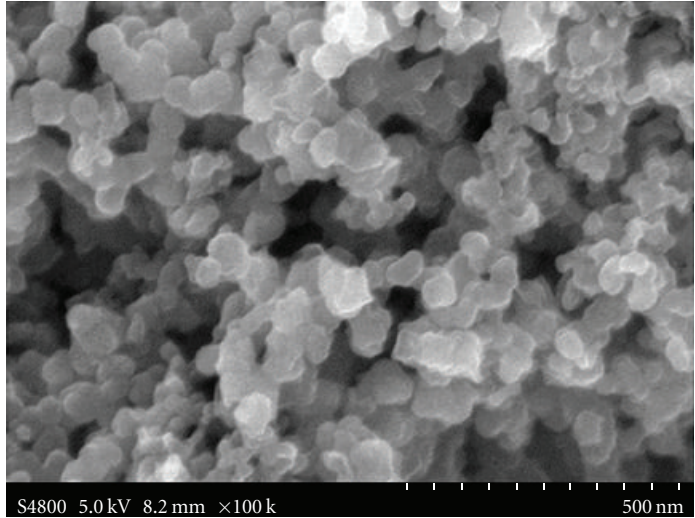

(b)

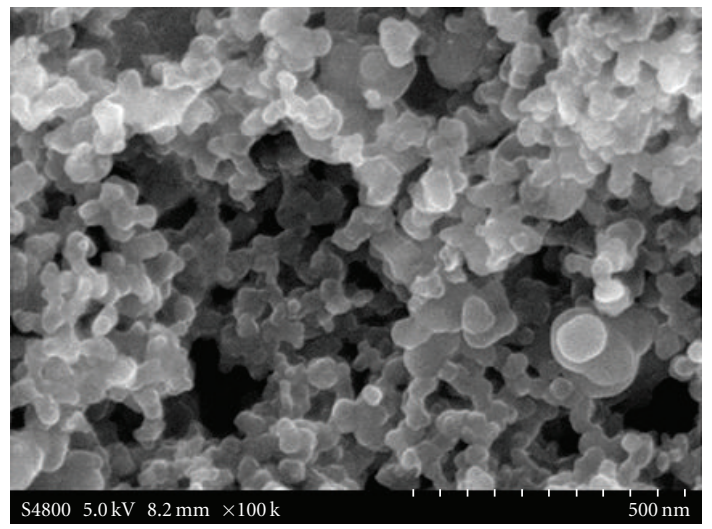

(d)

FIGURE 2: SEM micrographs of the deposited carbon on the internal wall of the reactor and the sand core (a) $6 \mathrm{~A}$, (b) $8 \mathrm{~A}$, (c) $14 \mathrm{~A}$, and (d) $20 \mathrm{~A}$.

which nanotube growth can occur. However, the excessively high temperature will cause sintering of the nanotubes. The temperature increases along with the arc discharge electric current elevates. Therefore, the $8 \mathrm{~A}$ current is ideal for the growth of nanotube.

A HRTEM image displayed in Figure 4 shows clearly that the deposited carbon on the inner wall of the reactor and the sand core is the turbostratic structure of graphite; that is, the deposited carbon is partially graphitized, not in a complete graphite phase. Figure 5 clearly shows well-graphitized layers of the multiwall nanotubes, the hollow shell morphology, and turbostratic structure deposited on the electrode ends. These CNTs consist of more than ten concentric carbon shells with a spacing of $0.34 \mathrm{~nm}$, which is consistent with that of graphite, only a little bit of carbonaceous material attached to its surface. The hollow shell structures can be considered as "giant fullerene": graphene layers are extended, their curvatures are related to pentagons, and no graphene edges can be observed.

3.2. X-Ray Diffraction Studies of Various Nanostructures. The measured diffraction patterns are shown in Figure 6. The inserted labels are Miller indices corresponding to the peaks of pure graphite. Only (002) peak appears in all patterns, (100) peak is visible in (e), and other weak peaks are invisible in all cases. The observed intensities for deposited carbon and their d-spacings seem to match those of the graphite$2 \mathrm{H}$ structure [11] with slightly expanded lattice parameters. The observed d-spacings of $0.3482,0.3440,0.3424,0.3421$, and $0.3419 \mathrm{~nm}$, corresponding to (a), (b), (c), (d), and (e), respectively, are wider than that of an ideal graphite crystal $(0.3354 \mathrm{~nm})$. This slight expansion in $\mathrm{d}_{002}$ is attributed to the turbostratic character in the carbon structure [5, 12-14]. In other words, graphitization degree of the carbon structure is inversely with d-spacing.

3.3. Raman Studies of Various Carbon Nanostructures. Raman measurements were performed using a $632.8 \mathrm{~nm} \mathrm{He}-$ $\mathrm{Ne}$ laser on the surface of the deposited carbon material. The Raman spectra of the deposited carbon (a) on the inner wall of the reactor and the sand core, and on the electrode ends of (b) $6 \mathrm{~A}$, (c) $8 \mathrm{~A}$, (d) $14 \mathrm{~A}$, (e) $20 \mathrm{~A}$, are shown in Figure 7. All spectra show mainly two Raman bands at 1328 and $1582 \mathrm{~cm}^{-1}$ corresponding to graphite's D- and G-bands, respectively. Generally speaking, for any carbon material, the peaks around $1328 \mathrm{~cm}^{-1}$ indicate the disorder in the sample due to carbon nanoparticles and the random orientation of the tubes, and the peaks around $1582 \mathrm{~cm}^{-1}$ are associated with the graphitic nature of the carbon materials [15]. Relative intensity ratio of D and G 


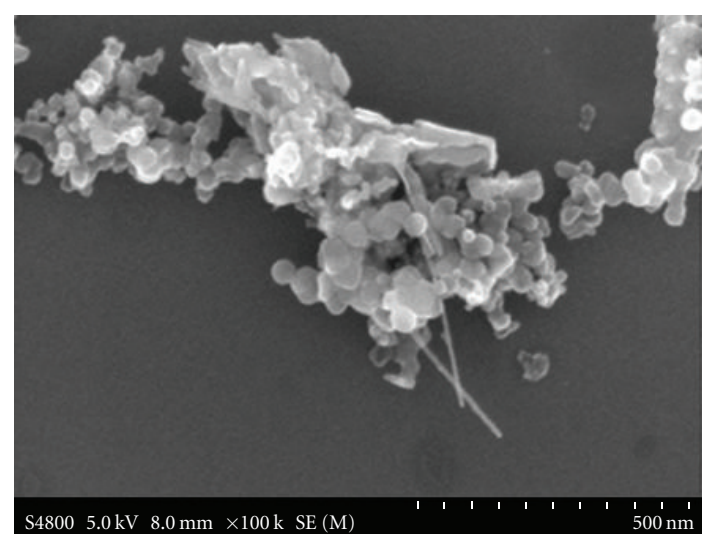

(a)

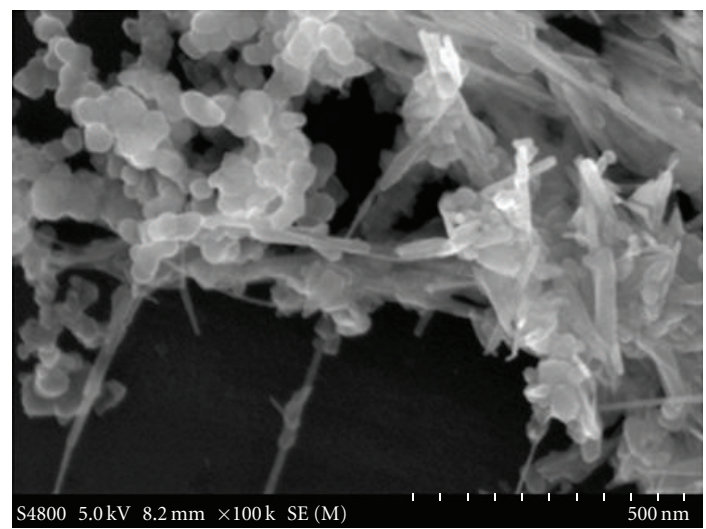

(c)

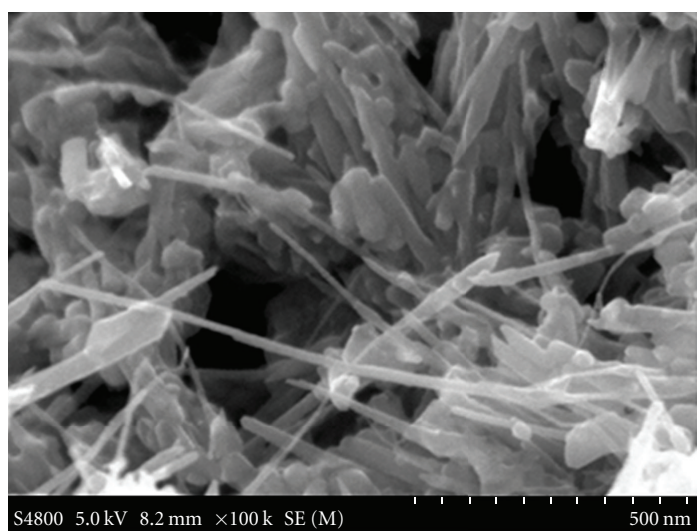

(b)

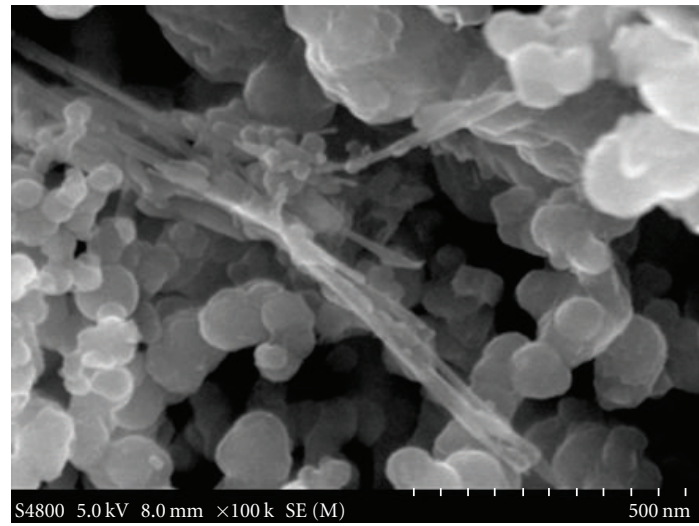

(d)

FIGURE 3: SEM micrographs of the deposited carbon on the electrode ends (a) $6 \mathrm{~A}$, (b) $8 \mathrm{~A}$, (c) $14 \mathrm{~A}$, and (d) $20 \mathrm{~A}$.

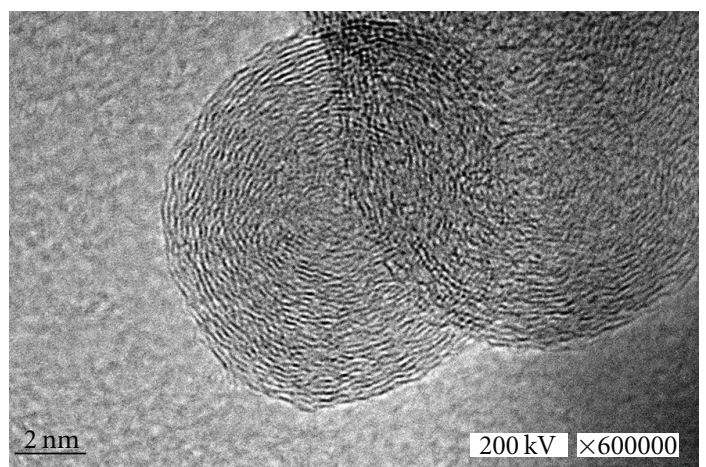

FIGURE 4: HRTEM micrographs of the deposited carbon on the inner wall of the reactor and the sand core.

peaks $\left(\mathrm{I}_{D} / \mathrm{I}_{G}\right)$ is a measure of the amount of disorder in the crystallinity [16]. Higher intensity of G band indicates the higher degree of crystallinity/graphitization. The value of $\mathrm{I}_{D} / \mathrm{I}_{G}$ decreases from 1.2 to 0.6 with the increasing arc discharge current. From these observations, it is concluded that the graphitization degree of the samples increases with the arc discharge current. The Raman results are nicely consistent with XRD analysis in which the crystallinity of graphitic sheets improves progressively as the arc discharge current increases.

3.4. Reaction Mechanism. A better understanding of the growth mechanism for nanotubes would help in the development of new efficient production methods. Many different theories have been proposed for the growth of nanotubes. Most theories rely on the presence of a metal catalyst [17-19]. However, for the growth of CNTs without catalysts, the majority of theories describe the formation of nanotubes from a carbon vapor comprised of carbon atoms, ions, and molecules within the plasma [20-22]. At the same time, other theories believe that a carbon vapor is not necessary for the growth of multiwalled nanotubes [3,23]. Nanotubes can be made by the heat treatment of disordered carbons.

In this paper, we present the following explanation for our observations. Firstly, carbon vapor comprising of carbon atoms, ions, and molecules was obtained from benzene by pyrolysis of plasma torch. Then, nucleation process results in the transformation of a molecular system to a particulate system. Thirdly, aggregation due to collisions of nanometersized particles (results of the nucleation process) forms 20-60 $\mathrm{nm}$ spherical particles (see Figure 3). The spherical particles were deposited on the inner wall of the reactor, sand core and electrode ends, which is of an aggregate of semispherical 


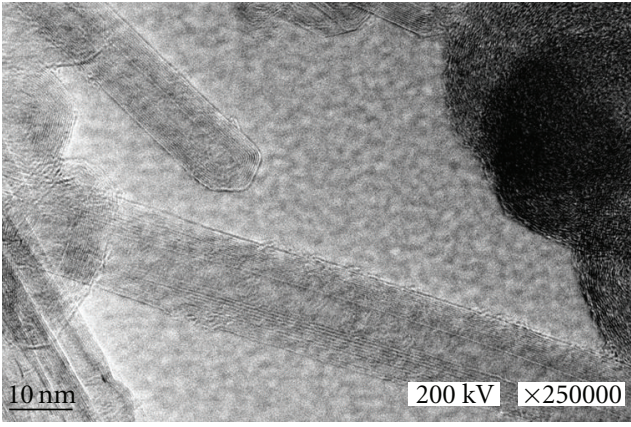

(a)

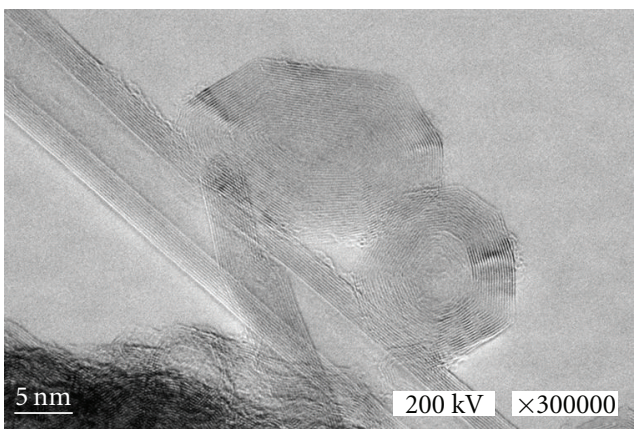

(b)

FIGURE 5: HRTEM micrographs of the deposited carbon on the electrode ends.

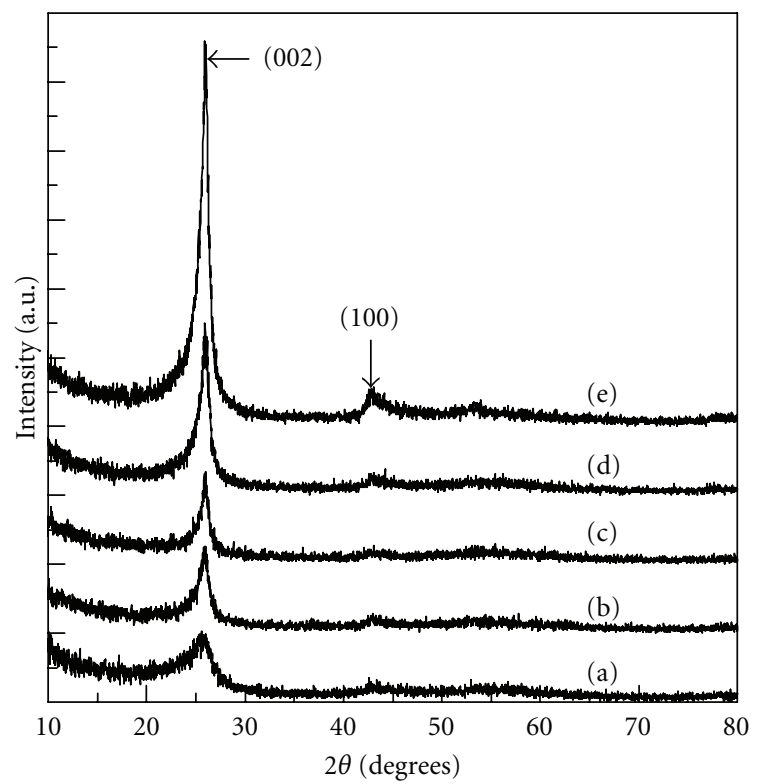

FIgURE 6: X-ray diffraction patterns of the deposited carbon (a) on the inner wall of the reactor and the sand core, and on the electrode ends of (b) $6 \mathrm{~A}$, (c) $8 \mathrm{~A}$, (d) $14 \mathrm{~A}$, and (e) $20 \mathrm{~A}$.

carbon-onion-like structures, built up by concentric carbonlayers around a defect-fullerene nucleus [24] (see Figure 5). After that, graphitization of the spherical particles deposited on the electrode ends begins with carbon self-diffusion by the heat treatment, leading to order in the planes of graphite

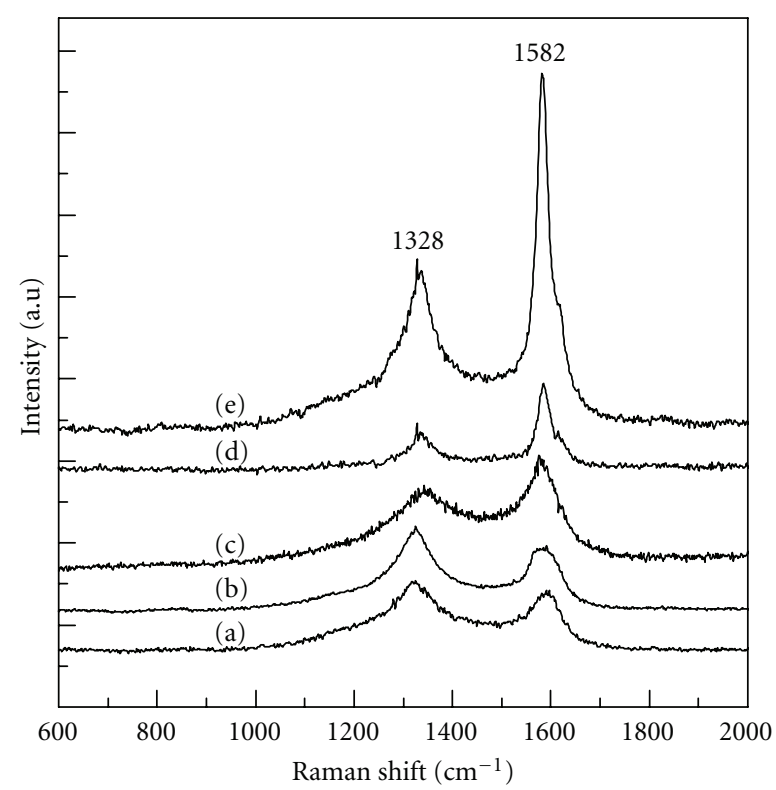

FIGURE 7: Raman spectra of the deposited carbon (a) on the inner wall of the reactor and the sand core, and on the electrode ends of (b) $6 \mathrm{~A}$, (c) $8 \mathrm{~A}$, (d) $14 \mathrm{~A}$, and (e) $20 \mathrm{~A}$.

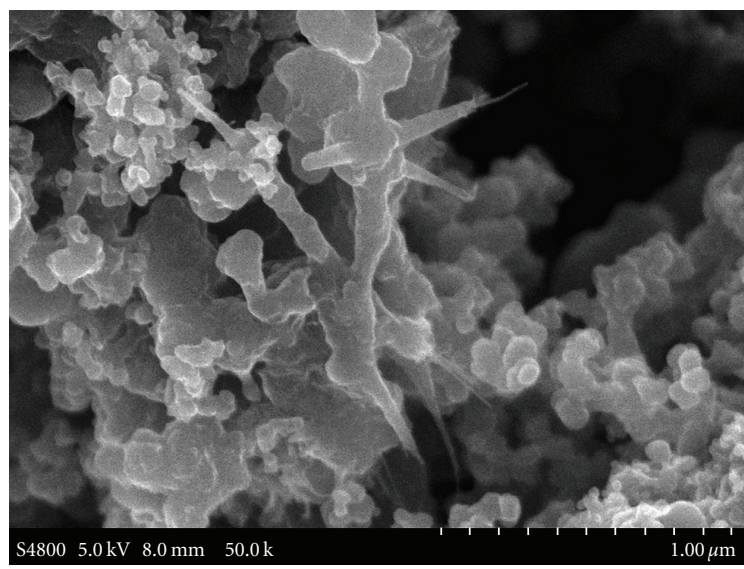

FIGURE 8: Schematic diagram of the formation of carbon nanotubes and agglomerate.

[25]. However, defects (pentagon, heptagon, and pentagonheptagon pairs) initially present in nongraphitizable carbons cannot be removed completely, which is necessary for the transformation of spherical particles to nanotubes [26-28]. The thermally generated tensile forces result in the formation of short nanotubes (no more than $1 \mu \mathrm{m}$ ), and agglomerate bulk formed by the melt of the particles simultaneously (see Figure 8).

\section{Conclusions}

Direct thermal decomposition of benzene was carried out using an AC arc discharge plasma process which is an environmentally favorable process.

The carbon particles deposited on the inner wall of reactor and the sand core exhibit a spherical particle with 
nanosized diameter. The diameter of the carbon particle varied from 20 to $60 \mathrm{~nm}$, and the mean diameter was about $30 \mathrm{~nm}$.

The carbon product deposited on the electrode ends is comprised of MWCNTs, aggregate carbon nanoparticles, and agglomerate carbon particles. The maximum yield content of CNTs occurs at an arc discharge current of $8 \mathrm{~A}$.

Further study is necessary in order to design a better plasma system and to produce larger quantities of nanotubes or carbon black.

\section{Acknowledgments}

The authors gratefully acknowledge Zhongqing Lin (Modern Experiment and Technology Center, Anhui University, China) for the SEM analysis, Yonglong Zhuang (Modern Experiment and Technology Center, Anhui University, China) for the HRTEM analysis. This work was supported by the National Natural Science Foundation of China (NSFC, nos. 20876100 and 20736004) the National Basic Research Program of China (973 Program, no. 2009CB219904).

\section{References}

[1] O. Vohler, G. Nutsch, G. Collin, F. von Sturm, and E. Wege, "Carbon," in Ullmann's Encyclopedia of Industrial Chemistry, vol. 6, pp. 281-292, Wiley-VCH, Weinheim, Germany, 2002.

[2] G. Kühner and M. Voll, Manufacture of Carbon Black in Carbon Black Science and Technology, Marcel Dekker, New York, NY, USA, 2nd edition, 1993.

[3] S. P. Doherty and R. P. H. Chang, "Synthesis of multiwalled carbon nanotubes from carbon black," Applied Physics Letters, vol. 81, no. 13, pp. 2466-2468, 2002.

[4] R. E. Haufler, J. Conceicao, L. P. F. Chibante et al., "Efficient production of $\mathrm{C} 60$ (Buckminsterfullerene), $\mathrm{C} 60 \mathrm{H} 36$, and the solvated buckide ion," Journal of Physical Chemistry, vol. 94, no. 24, pp. 8634-8636, 1990.

[5] S. Iijima, "Helical microtubules of graphitic carbon," Nature, vol. 354, no. 6348, pp. 56-58, 1991.

[6] A. Gutsch, M. Krämer, G. Michael, H. Mühlenweg, M. Pridöhl, and G. Zimmermann, "Gas-phase production of nanoparticules," KONA, no. 20, pp. 24-37, 2002.

[7] H. Murayama, S. Tomonoh, J. M. Alford, and M. E. Karpuk, "Fullerene production in tons and more: from science to industry," Fullerenes Nanotubes and Carbon Nanostructures, vol. 12, no. 1-2, pp. 1-9, 2004.

[8] J. Gonzalez-Aguilar, M. Moreno, and L. Fulcheri, "Carbon nanostructures production by gas-phase plasma processes at atmospheric pressure," Journal of Physics D, vol. 40, no. 8, article S16, pp. 2361-2374, 2007.

[9] K. Schmidt-Szalowski, T. Opalinska, J. Sentek et al., "Methane conversion into C2 hydrocarbons and carbon black in dielectric-barrier and gliding discharges," Journal of Advanced Oxidation Technologies, vol. 7, no. 1, pp. 39-50, 2004.

[10] P. J. F. Harris, "Solid state growth mechanisms for carbon nanotubes," Carbon, vol. 45, no. 2, pp. 229-239, 2007.

[11] I. Sanc, "Pattern: 00-041-1478, Graphite-2H, Polytechna," Foreign Trade Corporation, Panska, Czechoslovakia, ICDD Grant-in-Aid, 1990.

[12] L. Dobiasova, M. Stary, P. Glogar, and V. Valvoda, "Analysis of carbon fibers and carbon composites by symmetric X-ray diffraction technique," Carbon, vol. 37, no. 3, pp. 421-425, 1999.
[13] Y. Saito, T. Yoshikawa, S. Bandow, M. Tomita, and T. Hayashi, "Interlayer spacings in carbon nanotubes," Physical Review B, vol. 48, no. 3, pp. 1907-1909, 1993.

[14] K. Tohji, M. Sugano, A. Kasuya, Y. Nishina, Y. Saito, and H. Takahashi, "Resonant Raman scattering from single-walled nanotubes of small diameters," Applied Surface Science, vol. 144-145, pp. 657-662, 1999.

[15] R. Saito, G. Dresselhaus, and M. S. Dresselhaus, Physical Properties of Carbon Nanotubes, Imperial College Press, London, UK, 1998.

[16] M. Kumar and Y. Ando, "Single-wall and multi-wall carbon nanotubes from camphor-a botanical hydrocarbon," Diamond and Related Materials, vol. 12, no. 10-11, pp. 1845-1850, 2003.

[17] S. Iijima and T. Ichihashi, "Single-shell carbon nanotubes of 1 nm diameter," Nature, vol. 363, no. 6430, pp. 603-605, 1993.

[18] D. S. Bethune, C. H. Kiang, M. S. De Vries et al., "Cobaltcatalysed growth of carbon nanotubes with single-atomiclayer walls," Nature, vol. 363, no. 6430, pp. 605-607, 1993.

[19] C. Journet, W. K. Maser, P. Bernier et al., "Large-scale production of single-walled carbon nanotubes by the electric-arc technique," Nature, vol. 388, no. 6644, pp. 756-758, 1997.

[20] E. G. Gamaly and T. W. Ebbesen, "Mechanism of carbon nanotube formation in the arc discharge," Physical Review B, vol. 52, no. 3, pp. 2083-2089, 1995.

[21] T. Guo, P. Nikolaev, A. G. Rinzler, D. Tománek, D. T. Colbert, and R. E. Smalley, "Self-assembly of tubular fullerenes," Journal of Physical Chemistry, vol. 99, no. 27, pp. 10694-10697, 1995.

[22] J. C. Charlier, A. De Vita, X. Blase, and R. Car, "Microscopic growth mechanisms for carbon nanotubes," Science, vol. 275, no. 5300, pp. 646-649, 1997.

[23] A. A. Setlur, S. P. Doherty, J. Y. Dai, and R. P. H. Chang, "A promising pathway to make multiwalled carbon nanotubes," Applied Physics Letters, vol. 76, no. 21, pp. 3008-3010, 2000.

[24] V. I. Berezkin, "Fullerenes as nuclei of carbon black particles," Physics of the Solid State, vol. 42, no. 3, pp. 580-585, 2000.

[25] L. E. Jones and P. A. Thrower, "Influence of boron on carbon fiber microstructure, physical properties, and oxidation behavior," Carbon, vol. 29, no. 2, pp. 251-269, 1991.

[26] W. A. de Heer and D. Ugarte, "Carbon onions produced by heat treatment of carbon soot and their relation to the $217.5 \mathrm{~nm}$ interstellar absorption feature," Chemical Physics Letters, vol. 207, no. 4-6, pp. 480-486, 1993.

[27] P. J. F. Harris, S. C. Tsang, J. B. Claridge, and M. L. H. Green, "High-resolution electron microscopy studies of a microporous carbon produced by arc-evaporation," Journal of the Chemical Society, Faraday Transactions, vol. 90, no. 18, pp. 2799-2802, 1994.

[28] D. B. Geohegan, H. Schittenhelm, X. Fan et al., "Condensed phase growth of single-wall carbon nanotubes from laser annealed nanoparticulates," Applied Physics Letters, vol. 78, no. 21, pp. 3307-3309, 2001. 

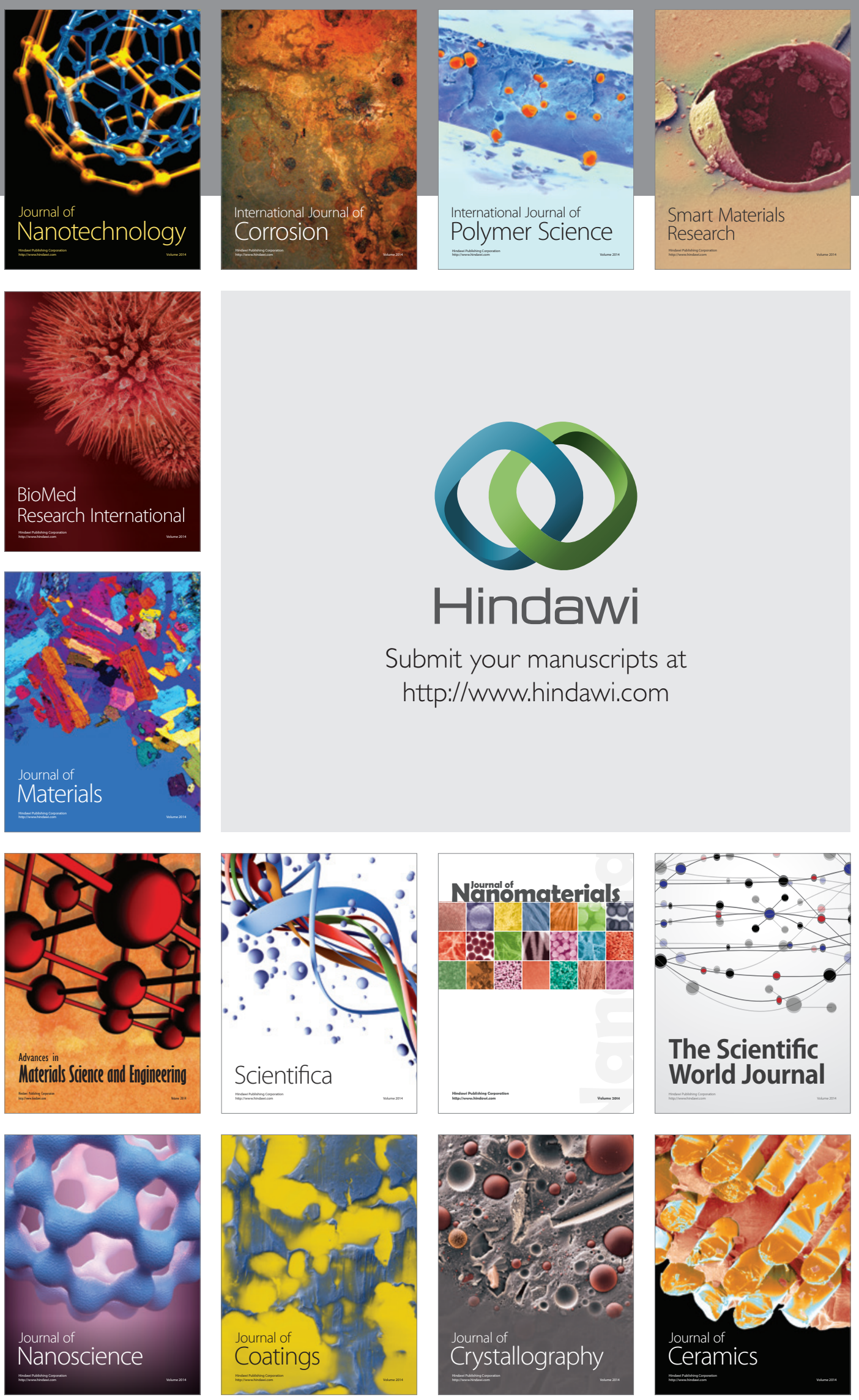

The Scientific World Journal

Submit your manuscripts at

http://www.hindawi.com

\section{World Journal}

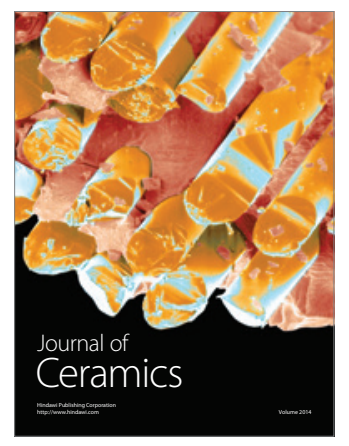

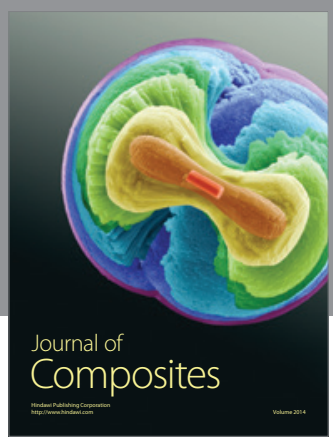
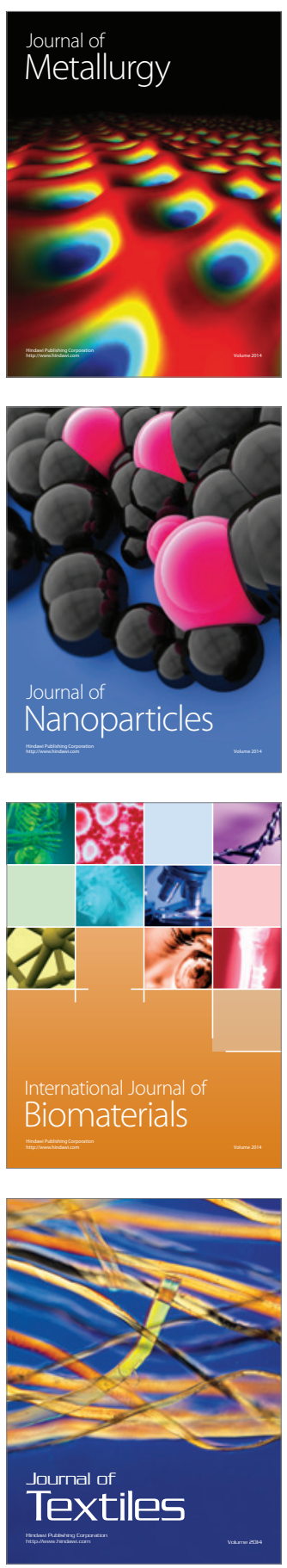